\title{
Charge Counter for Performing Active Charge-balance in Miniaturized Electrical Stimulators
}

\author{
L. Becerra-Fajardo ${ }^{1}$ and A. Ivorra ${ }^{1}$ \\ ${ }^{1}$ Universitat Pompeu Fabra/Department of Information and Communication Technologies, Barcelona, Spain
}

\begin{abstract}
Functional Electrical Stimulation (FES) has been explored in order to restore the capabilities of the nervous system in patients that suffer from paralysis. This area of research and of clinical practice greatly benefits from any technological improvement yielding miniaturization. In this regard, we recently proposed and demonstrated an innovative electrical stimulation method based on implanted microstimulators that operate as rectifiers of bursts of innocuous high frequency current supplied by skin electrodes, generating low frequency currents that are capable of stimulating excitable tissues. We envision flexible ultrathin implants (diameters $<300 \mu \mathrm{m}$ ) containing ASICs that have advanced capabilities, such as addressability and current control. As miniaturization is the main aim of this method, the use of bulky DC-blocking capacitors (e.g. $10 \mu \mathrm{F}$ ) to accomplish zero net charge injection and avoid electrochemical tissue and electrode damage is highly inconvenient. As an alternative, here we present an active charge-balance method based on the use of a digital charge quantifier, whose operation is inspired in the functioning of the tipping bucket rain gauge. The system monitors the charge injection, matching the charge injected in the cathodal phase, with the charge injected in the anodal phase, generating a biphasic current waveform that adapts itself to possible current source mismatches. We have implemented a prototype built with discrete components which uses a capacitor of only $100 \mathrm{pF}$ for the charge counter.
\end{abstract}

Keywords - FES, active charge-balance, dc-blocking capacitor, rectifier, miniaturization

\section{INTRODUCTION}

Electrical stimulation has been widely used, among other medical applications, for the restoration of muscle function in paralysis patients. This area of research and clinical practice is known as Functional Electrical Stimulation (FES). In order to stimulate muscles, electric currents are applied in the vicinity of motor nerves through electrodes by means of a pulse generator. Due to its location, these devices should ensure minimal invasiveness, which translates into minimal dimensions, and ease of implantation [1].

Currently, most electrical stimulation systems require complex surgeries for implantation, which limits their use to specific clinical applications. In the case of movement restoration, the use of a central stimulation unit that is wired to stimulating electrodes is not adequate for applications in which a large number of targets must be individually stimulated over large and mobile body parts. This restricts its use in patients that suffer of paralysis due to neurological disorders or spinal cord injuries. To solve this, an alternative approach has been explored: to develop a network of addressable single-channel wireless microstimulators that can be easily implanted (e.g. percutaneous injection). The microstimulators would be activated in coordinated patterns by an external controller. This has been tried in the past [2], but the implants obtained were stiff and too large or, in other words, too invasive.

When the composition of implantable electrical stimulators is analyzed, we can identify two elements that contribute drastically to their volume. The first element is the energy source, typically based on inductive coupling or batteries. For this matter, we recently proposed and demonstrated an alternative method of electrical stimulation [3] which accomplishes unprecedented potential for miniaturization (later further described). The second element is a mechanism for blocking DC currents in order to prevent deleterious electrochemical effects such as electrode corrosion and tissue damage [4]. Typically, these mechanisms are based on the use of a bulky DC-blocking capacitor which is passively or actively discharged. This capacitor needs to be large because it must integrate the total stimulation current [5]. When the objective is to minimize the implant's volume, or when multiple channels are required, this bulky element is of primary concern, and as a consequence, different active charge-balance approaches have been explored in order to avoid the DC-blocking capacitor [6].

Here we present an active charge-balance method consisting on a circuit that generates biphasic asymmetric current waveforms that adapt the pulse width of the anodic current by continuously monitoring the charge injected by the stimulating current source during the cathodal (negative current) and anodal (positive current) phases. Compared to other approaches for charge-balance, the proposed method minimizes complexity, and component count, and may offer higher robustness due to its semi-digital architecture. In addition, it uses a small capacitor that can be made external for higher tolerance to single fault conditions. 


\section{W IRELESS MICROSTIMULATORS BASED ON ELECTRONIC RECTIFICATION OF EPIDERMICALLY APPLIED CURRENTS}

Fig. 1. schematically illustrates the alternative method for performing electrical stimulation, which was proposed and in vivo demonstrated in [3]. The electronic implants rectify bursts of innocuous high frequency (HF) (e.g. $1 \mathrm{MHz}$ ) currents conductively supplied to the tissue of interest by remote electrodes so that low frequency (LF) currents are generated locally through the implants. These LF currents are able to stimulate local excitable tissue. We showed in [7] that the rectifiers can include advanced capabilities such as current control and addressability. In addition, we demonstrated in [8] that the HF bursts are innocuous, both in terms of heating and unintended electro-stimulation, and that portable systems will be feasible with present battery technologies.

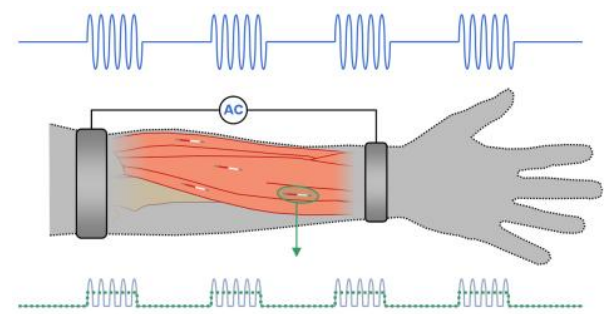

Fig. 1. The proposed method consists on using implants that act as rectifiers of innocuous AC current bursts. These currents are applied through external electrodes. The low frequency current densities generated by the implants are capable of locally stimulating tissue.

In order to operate, the method requires a minimum voltage drop between the implanted electrodes. Hence, we propose elongated implant bodies consisting on flexible and stretchable materials that match the mechanical properties of the surrounding tissue. Because of their suggested body and their intended functionality, these implants were coined "Electronic Axons" (eAXONs).

We envision ultrathin eAXONs (diameter $<300 \mu \mathrm{m}$ ) containing ASICs with advanced capabilities, such as communication, control and sensing. The implants will form a complex network as a large number of devices will be percutaneously located in the target tissue. The network will be controlled by an autonomous portable external system which delivers the innocuous HF currents. In this way, the system will be able to execute complex stimulation patterns such as those that would be required for fine movement restoration in paralysis patients.

As miniaturization is a key objective, eAXONs will need the smallest possible electronic body (i.e. minimization of the number and sizes of components used). In the first assayed architectures [9], we used biphasic current waveforms complemented by a $10 \mu \mathrm{F}$ DC-blocking capacitor to prevent electrode and tissue damage. However, $10 \mu \mathrm{F}$ capacitors are too large for the envisioned ultrathin eAXONs. To solve this, we propose a new method for active charge-balance that avoids the use of capacitors of more than $100 \mathrm{pF}$ capacitance.

\section{Proposed method for active Charge-Balance}

The proposed eAXON circuit architecture (later described) consists of two independent current sources which generate low frequency biphasic currents. As mismatches between the current sources may appear, we propose: 1- to monitor the charge injected during the cathodal phase using a small capacitor (e.g. $100 \mathrm{pF}$ ), and then 2- to match this charge during a pulse-width-adapted anodal phase, generating an asymmetric biphasic waveform (Fig. 2). In other words, we propose a system able to quantize and match the amount of charge injected in every phase of the biphasic waveform, avoiding the use of large capacitors.

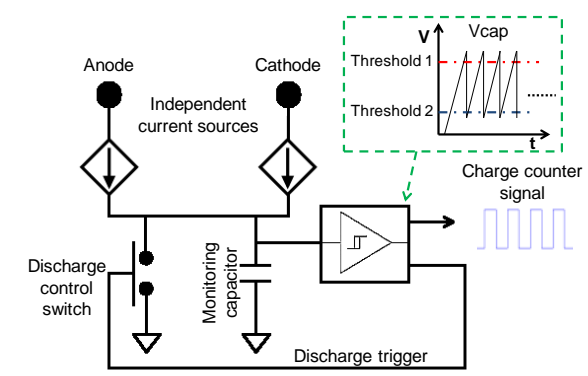

Fig. 2. Proposed circuit architecture for monitoring the electrical charge injection. The circuit is capable of discharging a small capacitor, and generating a charge counter signal for the control unit.

The proposed circuitry (Fig. 2), whose architecture was inspired by the tipping bucket rain gauge, consists of a monitoring capacitor that charges when an independent current source is enabled. The voltage in the capacitor is compared to a pair of thresholds, in order to control the amount of charge injected by the current source. This is accomplished by a comparator circuit with hysteresis (i.e. Schmitt trigger). The output of this circuit is then connected to a switch that shorts the capacitor, inducing its discharge. In addition, this circuit generates a charge counter signal that is read by the control unit, to sum the charge accumulated by the capacitor in a stimulation phase.

The process starts when the cathodal phase is enabled. At the end of this stage, the charge count is registered. As the capacitor used is small, hundreds of discharges are counted per phase. When the control unit enables the second current source, generating the anodal phase, the process repeats. A number of charge counts (proportional to the charge injected by the current source) are registered by the control unit 
and are matched to the charge count recorded during the cathodal phase. When the same number of charge counts is reached, the anodal phase is disabled, accomplishing a zero net charge injection.

In short, the fixed pulse width of the cathodic current generates a specific number of charge counts, which in turn fixes the number of charge counts that must be generated by the anodic current, creating a charge-balanced asymmetric biphasic current waveform.

\section{COMPLETE CIRCUIT ARCHITECTURE}

The circuit architecture for an eAXON demonstrator based on discrete components is shown in Fig. 3. Seven blocks can be identified: 1- two electrodes that pick-up the high frequency AC current that flows through the tissues and that deliver LF currents for stimulation, 2- a full bridge rectifier and a regulation subcircuit, which electrically feed the control unit and the current sources, 3- an amplitudeshift keying (ASK) demodulator 4- a power switch that disconnects the regulation subcircuit and the demodulator when the stimulating pulses are generated, 5- two switchable current sources that generate biphasic currents, 6- a charge injection counter, and 7- the control circuit.

The control circuit is based on one of the smallest microcontrollers commercially available $(2 \mathrm{~mm} \times 2 \mathrm{~mm}$ footprint): the ATtiny 10 by ATMEL Corp. Commands (e.g. address of the stimulator to be activated and the pulse width of the cathodal phase) are transmitted from the external system by means of amplitude-shift keying at a rate of 20 kbauds on a $1 \mathrm{MHz}$ sinusoidal current. The current sources are based on a zener diode that fixes a voltage on the base of a NPN transistor, therefore defining the current flowing through the collector and the emitter.

The implemented power switch avoids the flow of stimulating current through the regulation and the demodulation subcircuits when the current sources are enabled. This switch is designed with a combination of NPN and PNP transistors and a NOR logic gate (SN74LVC1G27 by Texas Instruments, Inc.), which is in turn governed by control signals 1 and 2 (CS1 and CS2).

The charge counter uses a custom made Schmitt trigger based on an operational amplifier (AD8605 by Analog Devices, Inc.), and a $100 \mathrm{pF}$ capacitor. Due to the speed of charge of the small monitoring capacitor, the selectable Thresholds 1 and 2 (Fig. 2) were set at $1.8 \mathrm{~V}$ and $1.2 \mathrm{~V}$ respectively, as they give enough time for the comparator to trigger a charge count and the control unit to read it. The discharge switch was designed using a NPN transistor (BC817-25LT1 by ON Semiconductor Corp.).

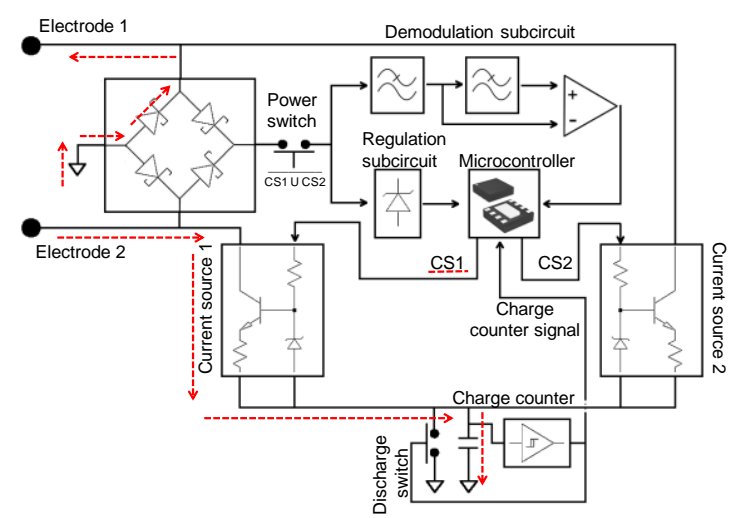

Fig. 3. Circuit architecture of the demonstrator. The dashed line shows the flow of the rectified current when the control signal 1 (CS1) activates the current source 1 . The charge monitor controls the discharge switch and generates a charge counter signal that is read by the control unit.

\section{v. Demonstration}

The setup shown in Fig. 4 was built to demonstrate the active charge-balance method proposed. The circuit is drawn in black and a simplified circuit for the rest of the system (i.e. external generator and tissue impedances) is represented in gray. The electrodes of the circuit are represented with nodes E1 and E2, and their impedances are neglected for simplicity. The resistances were chosen to coarsely represent muscle tissue impedances for a $3 \mathrm{~cm}$ eAXON according to Finite Element Method simulations (not reported here).

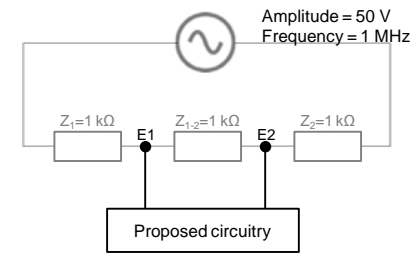

Fig. 4. Setup used to demonstrate the capability of the system to accomplish active charge-balance with the method proposed.

HF bursts were transmitted in order to generate biphasic asymmetric current pulses at a rate of $20 \mathrm{~Hz}$. At first, control signal 1 (CS1) activates a current source for $100 \mu \mathrm{s}$. This current source generates a cathodic current while charging the monitoring capacitor. As explained in Section IV, during this pulse the charge counter circuit automatically discharges the capacitor, and generates a charge count signal that is read by the microcontroller. At the end of the pulse, the number of discharges is saved for the anodic pulse process. In-between pulses, an interphase dwell of $10 \mu \mathrm{s}$ is included. To generate the anodic pulse, the second current source is enabled by the control signal 2 (CS2). The control unit reads the signal generated by the charge counter 
circuit, and matches the number of counts with that obtained in the cathodic pulse, therefore matching the charge injected by each phase of the stimulation.

Fig. 5 shows the results obtained with the proposed setup. Using a battery powered oscilloscope (TPS2014 by Tektronix, Inc.), we saved the low frequency components of the current flowing through the circuit. This was done recording the voltage drop across a RC low pass filter made with the parallel combination of a $10 \Omega$ resistor and $2.2 \mu \mathrm{F}$ capacitor (cutoff frequency $=7.2 \mathrm{kHz}$ ).
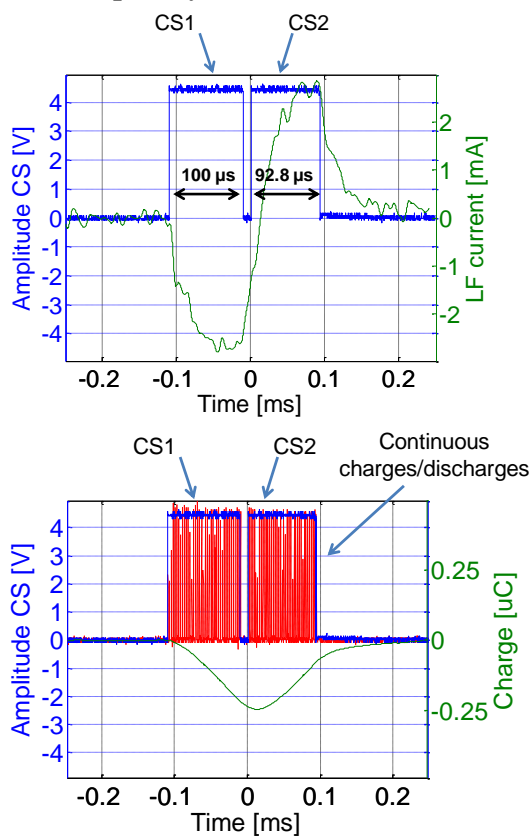

Fig. 5. Low frequency current and its corresponding charge when the system applied a cycle of cathodic and anodic current pulses. The behavior of the current sources is controlled by signals CS1 and CS2.

The results show that the current sources implemented were able to generate a low frequency current peak value of $2.7 \mathrm{~mA}$ in the cathodal phase and a peak value of $2.8 \mathrm{~mA}$ in the anodal phase, which is enough to electrically stimulate nerves [10]. The calculated charge shows that the circuit was able to inject over $0.22 \mu \mathrm{C}$ in the cathodal phase of the stimulation and that this charge was balanced during the anodal phase.

Fig. 5 also shows the continuous capacitor voltage charges/discharges. A simple algorithm showed that the number of capacitor charges of the anodal phase matched those of the cathodal phase. As there is a slight mismatch between current sources, the system was capable of adjusting the pulse width of the anodic phase, enabling the second current source for $92.8 \mu \mathrm{s}$.

In short, the method proposed is capable of stimulating excitable tissue and is able to generate a charge-balanced biphasic asymmetric current waveform that avoids the use of large profile capacitors typical of DC-blocking capacitor methodologies.

\section{ACKNOWLEDGMENT}

AI's research is supported by a Ramón y Cajal fellowship from the Spanish government and a Marie Curie grant (IRG 256376) from the European Commission. LBF's research is supported by a scholarship from the UPF.

\section{Conflict of InTEREST}

The authors declare that they have no conflict of interest.

\section{REFERENCES}

1. Peckham P H, Ackermann D M (2009) Chapter 18 - Implantable Neural Stimulators. Neuromodulation, Academic Press, San Diego

2. Schulman, J. H. (2008) The Feasible FES System: Battery Powered BION Stimulator. Proc IEEE. vol. 96, no. 7, 2008, pp. $1226-1239$

3. Ivorra, A. (2011) Remote Electrical Stimulation by Means of Implanted Rectifiers. PLoS One 6:p.e23456

4. Cogan, S. F (2008) Neural Stimulation and Recording Electrodes. Annu Rev Biomed Eng 10:275-309

5. Ortmanns M (2007) Charge Balancing in Functional Electrical Stimulators: A Comparative Study, IEEE International Symposium on Circuits and Systems, 2007, pp. 573-576

6. Ghovanloo M, Najafi K (2007) A wireless implantable multichannel microstimulating system-on-a-chip with modular architecture. IEEE Trans Neural Syst Rehabil Eng 15:449-57

7. Becerra-Fajardo L, Ivorra A (2012) Proof of Concept of a Stimulator Based on AC Current Rectification for Neuroprosthetics Cong. Anual Soc. Esp. Ing. Bioméd. CASEIB, San Sebastián, Spain, 2012, pp 76

8. Ivorra A, Becerra-Fajardo L (2013) Wireless Microstimulators Based on Electronic Rectification of Epidermically Applied Currents: Safety and Portability Analysis, 18th IFESS Annual Conference, 2013, pp. 213-216

9. Becerra-Fajardo L, Ivorra A (2014) Towards Addressable Wireless Microstimulators Based on Electronic Rectification of Epidermically Applied Currents, Proc 36th Annual International Conference of the IEEE Engineering in Medicine and Biology Society, 2014, no 1306

10. Sauter A R, Dodgson M S, Kalvøy H et al. (2009) Current Threshold for Nerve Stimulation Depends on Electrical Impedance of the Tissue: A Study of Ultrasound-Guided Electrical Nerve Stimulation of the Median Nerve. Anesth Analg 108:4

Author: PhD. Antoni Ivorra

Institute: Universitat Pompeu Fabra

Street: Carrer Roc Boronat, 138 (51.008) - 08018

City: Barcelona

Country: Spain

Email: antoni.ivorra@upf.edu 